\title{
Extraforaminal lumbar disc herniation at two contiguous intervertebral levels
}

\author{
Hisatoshi Baba, Yasuhisa Maezawa, Nobuaki Furusawa, Kenzo Uchida, Hideo Kawahara and Yasuo Kokubo \\ Department of Orthopaedic Surgery, Fukui Medical School, Shimoaizuki 23, Matsuoka, Fukui 910-11, Japan
}

\begin{abstract}
We describe two unusual surgical cases who presented with extraforaminal lumbar disc herniation that occurred at two adjacent vertebral levels simultaneously and unilaterally. Magnetic resonance imaging and selective nerve root infiltration followed by radiculography helped to outline the herniated disc material. Lateral fenestration and microsurgical foraminal widening of the affected vertebral levels allowed a complete and safe relief of the compressed nerves.
\end{abstract}

Keywords: lumbar radiculopathy; extraforaminal intervertebral disc herniation; microsurgery; foraminotomy, double-level disc herniation

\section{Introduction}

Extraforaminal intervertebral disc herniation is an uncommon cause of lumbar radiculopathy. ${ }^{1,2}$ Coexisting intraspinal pathology, spinal canal stenosis and/or disc herniation, may obscure the diagnosis of farlateral entrapment of the lumbar spinal nerve. However, awareness of this extracanalicular pathology has increased significantly by the use of high resolution computed tomography, magnetic resonance imaging, (MRI), and selective nerve root infiltration technique followed by radiculogrpahy. ${ }^{2,3}$ Different surgical approaches ${ }^{4-7}$ have been reported but it is essential to minimise surgical invasiveness and avoid resection of excess amount of osteoligamentous tissue in order to maintain lumbar spine mobility.

Multiple extraforaminal lumbar disc herniation at different vertebral levels is rare. In this report, we describe two surgical cases of double-level extraforaminal lumbar disc herniations, and stress the importance of neuroimaging in the diagnosis as well as emphasise certain surgical considerations.

\section{Case presentations}

Case 1

A 49-year-old woman was admitted to our hospital complaining of intractable pain of radicular nature in her left thigh and lower leg. She was unable to lie supine, sit, or to walk due to pain. She was otherwise healthy and had no significant past medical history, and the family history was negative.

Neurological examination identified painful dysesthesia at L4 and L5 left dermatomes. The straight leg raising test (SLRT) was positive on the left at 30 degrees. SLRT

Correspondence: H Baba on the right leg at 50 degrees provoked radicular pain on the left leg (positive crossed-SLRT). The Kemp test (lateral bending test during standing) was positive on the left, however, right-side bending of the lumbar spine caused marked thigh and leg pain on the left side. Manual muscle testing showed a limited weakness of the quadriceps muscle (grade 4/5). Deep tendon reflex showed a slightly hypoactive patellar tendon reflex. No pathological reflex or clonus was present. Bladder function was normal. Lumbar spine radiographs showed degenerative spondylosis at L3-4, L4-5 and L5-S1 vertebral levels, without any osteolytic appearance indicative of metastasis or infection. The anteroposterior film showed slight lumbosacral scoliosis (Cobb's angle 18 degrees) with left-sided convex curvature. MRI scan showed a slightly bulged intervertebral disc at these levels in the sagittal display but, more significantly, superimposed left L4-5 and L5-S1 extraforaminal disc herniations (Figure 1a). Selective nerve root infiltration using $2 \%$ lidocaine $(2 \mathrm{ml}$ for each spinal nerve) followed by radiculography demonstrated caudally displaced L4 spinal nerve at the foraminal exit ${ }^{8}$ and a markedly swollen L5 spinal nerve (Figure 1b). These findings were suggestive of a 'double-level' (L4-5 and L5-S1 levels) extraforaminal lumbar disc herniation.

The patient underwent microsurgical discectomy through a lateral fenestration (partial laminotomy of the inferior articular process) $)^{7,9}$ at L4-5 and L5-S1 levels. Microsurgical exploration showed a fragmented herniated disc at the exiting zone of the neuroforamen at L4-5 level and an extrusion-type hernia at the same zone of the L5-S1 intervertebral foramen (Figure 1c). The L4 and L5 spinal nerves were completely relieved from compression after discectomy without a concomitant spinal fusion involving these levels. The patient was ambulatory 5 days after the operation and was discharged 2 weeks later. At 2.5-year follow-up, she was 


Multiple extraforaminal lumbar disc herniations
$\mathrm{H}$ Baba et al

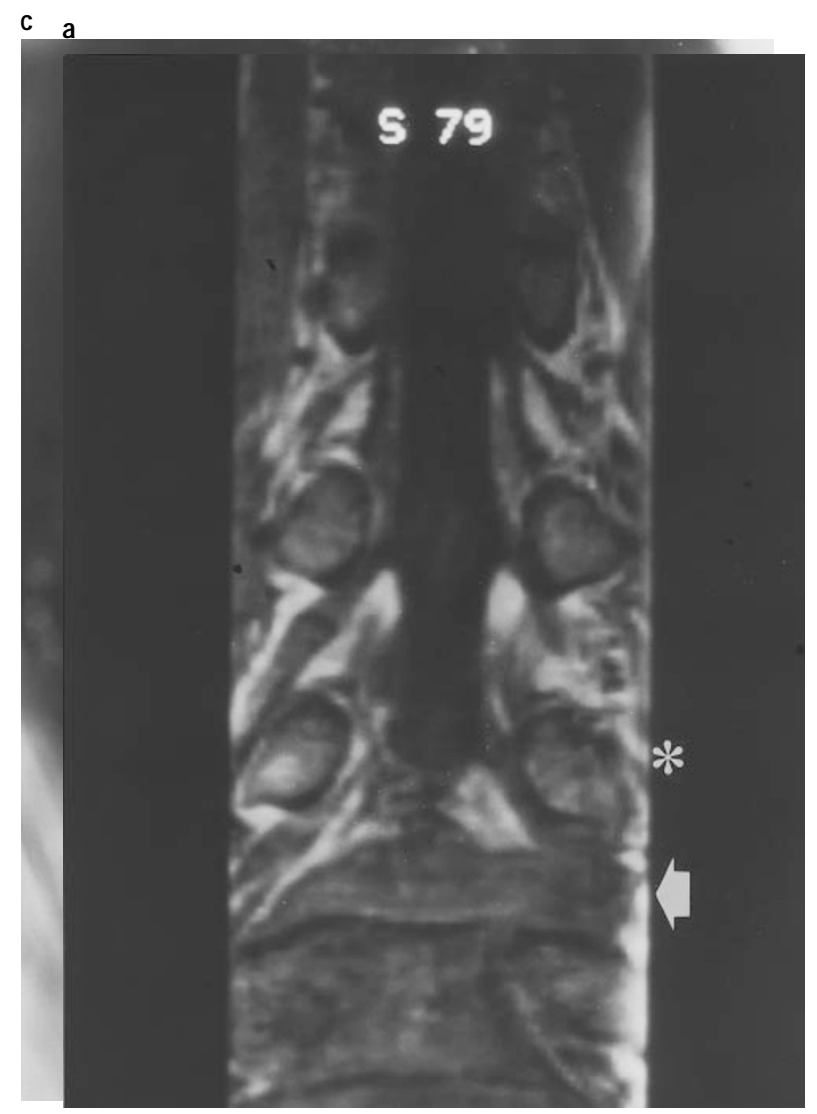

b

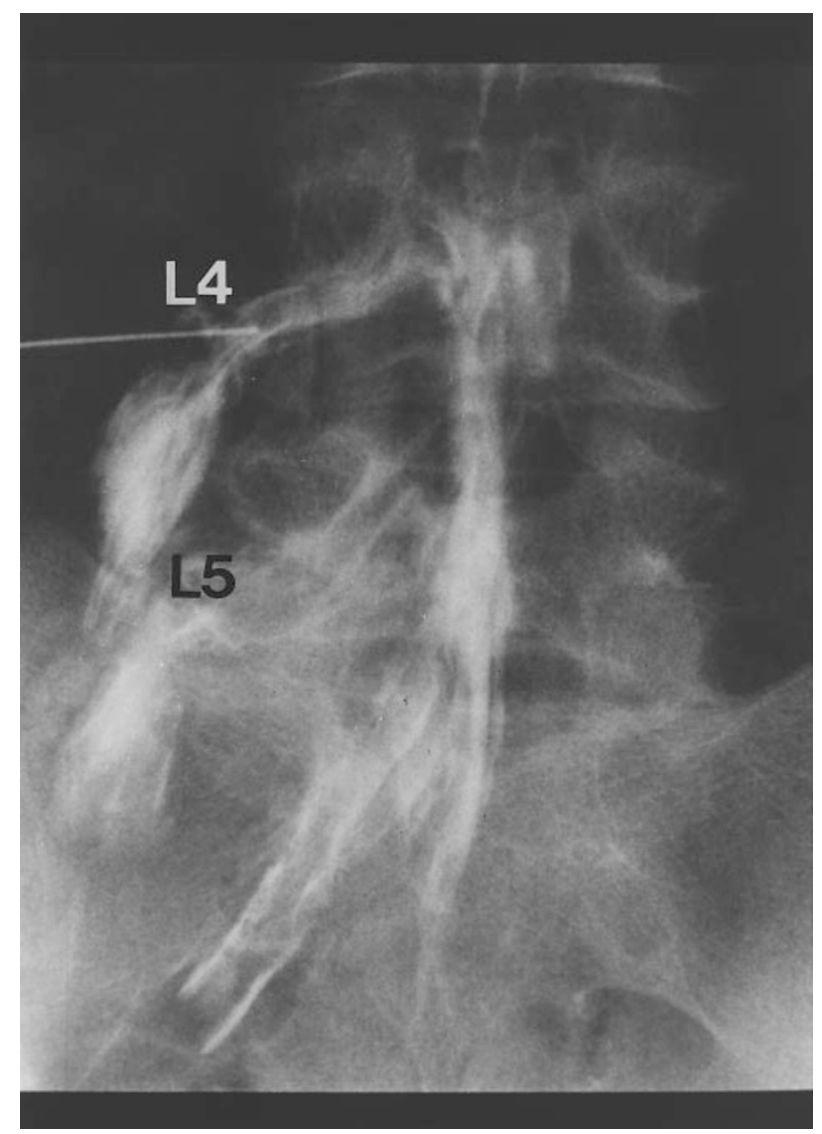

C

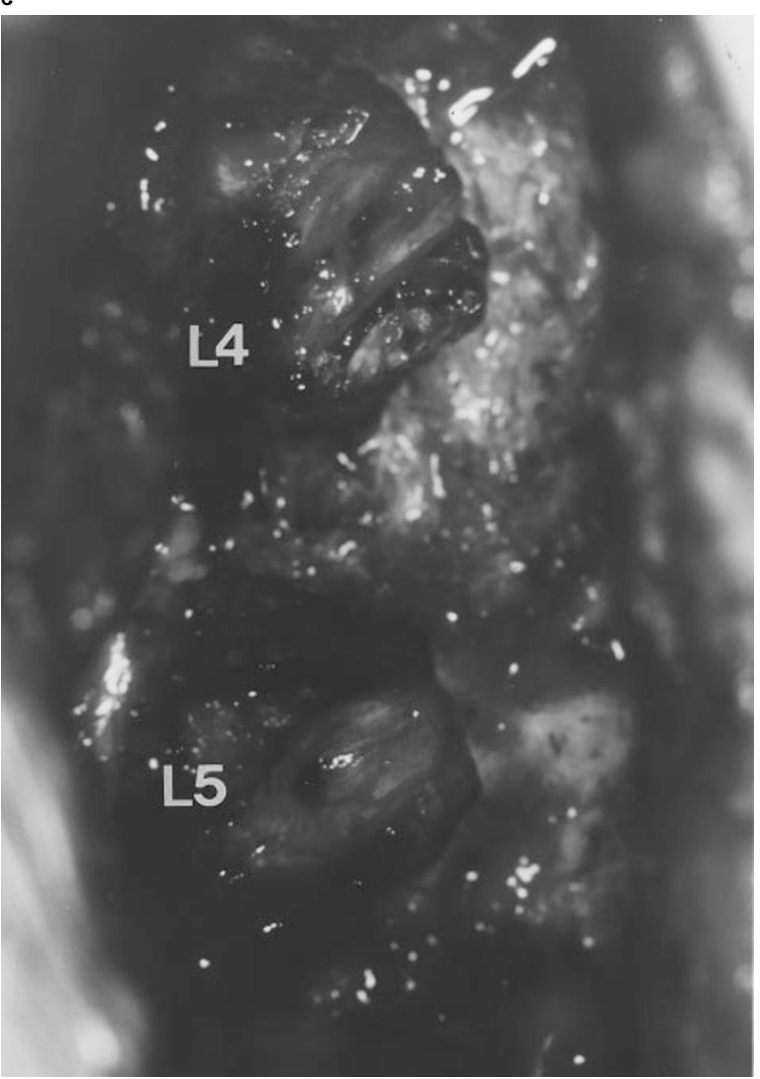

Figure 1 (a) Reconstructed magnetic resonance image in the coronal plane of case 1. A fragmented herniated disc (asterisk) at L4-5 level and an extruded type disc hernia (arrow) at L5-S1 level on the left side. (b) Selective radiculography of case 1 demonstrating caudally displaced L4 (L4) and significantly swollen L5 (L5) spinal nerves on the left side. (c) Microscopic photograph taken intraoperatively showing decompressed L4 (L4) and swollen L5 (L5) spinal nerves 


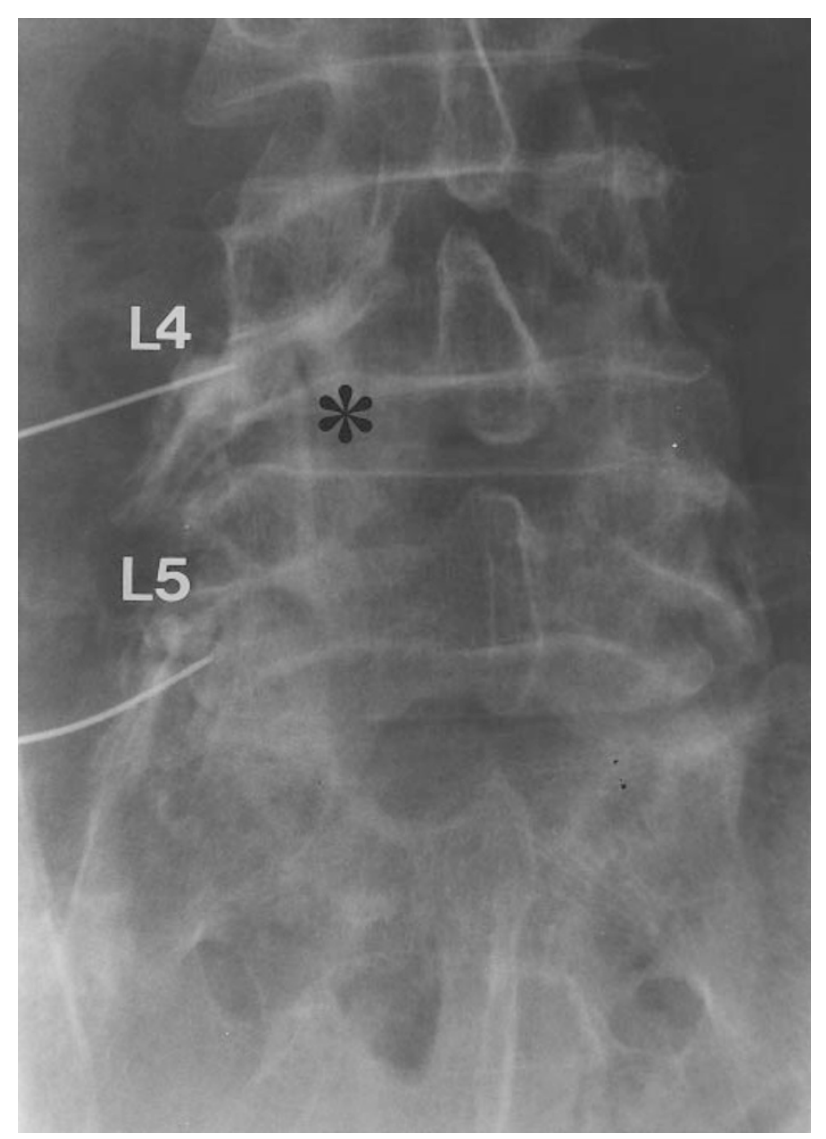

Figure 2 Selective radiculography on the left side of case 2 showing abnormally round shadow (asterisk) suggestive of an extruded hernia to compress the L4 spinal nerve (L4) and round and deviated L5 spinal nerve (L5)

doing very well as a school teacher without complaining of lower back pain and leg pain. Radiographs showed no instability ${ }^{3}$ at L4-5 and L5-S1 levels.

\section{Case 2}

This 63-year-old man complained of gluteal and leg pain of radicular nature on the left for about 2 weeks before presentation. Walking for approximately $3 \mathrm{~min}$ (20-30 metres) significantly increased radicular pain and forced him to rest. The past medical history and family history were negative.

On presentation, Kemp's sign was remarkable on the left and SLRT was positive at about 40 degrees on the left side. The femoral nerve stretch test was also positive on the left. A Valsalva manoeuvre exacerbated radicular pain. Neurological examination showed painful dysesthesia at the left L4 dermatome and hypesthesia at L5 nerve area on the left. The patellar tendon reflex was diminished on the left, and the strength of the left rectus femoris muscle was assessed as grade $4^{+} / 5$. Radiographs of the lumbar spine demonstrated spondylosis at L3-4, L4-5 and L5-S1, while MRI displayed a narrow neural foramen at L4-5 level and a suspected intraforaminal herniated disc at the same level. Selective nerve root infiltration with radiculography technique was thus indicated and demonstrated that the spinal nerves of L4 and L5 were compressed by an abnormal mass, suggestive of intraforaminal hernia at L4-5 neuroforamen and extraforaminal hernia at the exit region of the L5-S1 foramen (Figure 2).

The patient underwent microsurgical foraminal widening ${ }^{3}$ followed by discectomy at L4-5 level, and excision of an extruded extraforaminal hernia after lateral foraminotomy at L5-S1 level on the left side. On the left side of L4-5, the L4 spinal nerve was impinged by both the extraforaminal bulged disc and extruded hernia mass within the foramen, just ventral to the L4 ganglion. On the left side of L5-S1, the extraforaminal extruded hernia compressed the L5 spinal nerve at the exiting zone of the foramen. Both hernias were completely excised without removing much of the inferior articular process of L4 and the sacral area on the left, requiring no spinal fusion technique. The patient reported complete amelioration of symptoms soon after the operation and was discharged 8 days postoperatively. The patient has been doing well at 11month follow-up without low back and leg pain.

\section{Discussion}

Lumbar radiculopathy is infrequently caused by extraspinal (intra- and extraforaminal) herniation of an intervertebral disc. The frequency of disc degeneration may increase with ageing, similar to that of intraspinal pathology. Between 1987 and 1996, a total of 778 lumbar disc hernias (683 patients) were excised by one of the authors (HB) in our Medical Centre and affiliated institutions. Of those, $48 \quad(6 \%)$ hernias occurred primarily at the intraforaminal $(n=19)$ and/ or extraforaminal $(n=39)$ areas in 46 patients $(35$ men and 11 women; average age, 46 years; range, 39-68 years), including the present cases. The distribution of these hernias according to the affected vertebral level was one hernia at L2-3 level, $22(46 \%)$ at L3-4 level, $19(40 \%)$ at L4-5 level, and $6(13 \%)$ at L5-S1 level. Our experience, however, is not comparable with that reported by other centres, ${ }^{1,2,6}$ most likely due to differences in surgical indications. In general, extraforaminal and/or intraforaminal disc herniation may constitute only $5-12 \%$ of lumbar disc herniation, ${ }^{2.6}$ however, to our knowledge, no report has described a 'double-level' extraforaminal herniation as reported in the present communication. Though comparative data are not available to describe the prevalence of this 'double-level' lesion, the rate appears to be low.

The clinical presentation of this 'double-level' extraforaminal herniation commonly involves more severe signs and symptoms compared with those of single-level involvement. In patients with intractable pain extending over a large area of the leg, neurological determination of the level(s) of symptomatic nerve root and/or the cauda equina may be 
hindered. Hence, repeated neurological examinations and tests are important. In intra- and extraforaminal lumbar disc herniation, the nerve root (spinal nerve) is compressed one-level above the hernia, and the severity of clinical symptoms and signs is often worse than that of intraspinal pathology, primarily due to the compression of the nerve root ganglion. During radiological diagnosis, as Epstein et $a l^{6}$ have correctly recommended, one should be extremely cautious particularly for ruling out intraspinal compressive lesions. Obviously, the presence of a stenotic lumbar spinal canal and/or intraspinal bulged disc in elderly patients may not allow an accurate diagnosis of a true extra- and/or intraforaminal herniation. Moreover, coexisting foraminal stenosis ${ }^{3,10,11}$ must be carefully evaluated. In case 1 described in this study, coronal reconstruction of MR images was helpful to visualise the relationship between the symptomatic spinal nerves and herniated discs (Figure 1a). Selective nerve root infiltration and radiculography were additionally useful diagnostic tools for identifying the exact site and degree of neural compression (Figures $1 \mathrm{~b}$ and 2). A combination of these neuroimaging techniques may reduce the rate of undiagnosed or misdiagnosed extraand/or intraforaminal disc herniation but, more importantly, we suggest that a careful and thorough neurological examination is an essential part of suspecting the 'double-level' lesion.

The surgical options include a variety of approaches, such as facetectomy, hemilaminectomy, and lateral fenestration with or without medial foraminotomy..$^{2-5,7,9,12-16}$ As we have described previously, ${ }^{3}$ excessive bone resection and unnecessary fusion should be avoided during excision of doublelevel hernias as well as decompression of the affected spinal nerve. Facetectomy or hemilaminectomy approach with or without osteoplastic procedures require spinal fusion at three segments involving the affected levels, although these approaches usually provide a

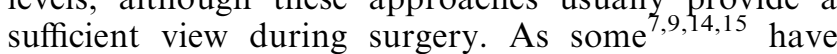
indicated, extraforaminally herniated discs should and can be resected via the lateral fenestration approach. Even in a case with a 'double-level' extraforaminal hernia, the lateral approach is to be considered. Furthermore, in a case with an additional intraforaminal herniation of the disc, as Autrique et $a l^{12}$ described, a combination of medial and lateral fenestration is suggested for complete resection of the herniated mass. On the other hand, in patients with additional narrowing of the neural foramen as in case 2 in the present study, microsurgical widening of the nerve root canal ${ }^{3}$ should also be taken into consideration during safe resection of intra- and extraforaminal herniation of the disc.
In conclusion, we have presented two cases with a unilateral 'double-level' extraforaminal intervertebral disc herniation of the lumbar spine, and have demonstrated the significance of a precise neurological examination and of detailed neuroimaging. We also outlined our recommendations for the most appropriate surgical approach to minimise the chance of multi-level spinal fusion and maximising a safe resection of the lesions compressing the affected spinal nerves.

\section{References}

1 Macnab I. Negative disc exploration: an analysis of the causes of nerve root involvement in sixty-eight patients. J Bone Joint Surg Am 1971; 53: 891 - 903 .

2 Kunogi J, Hasue M. Diagnosis and operative treatment of intraforaminal and extraforaminal nerve root decompression. Spine 1991; 16: $1312-1320$.

3 Baba $\mathrm{H}$ et al. Microsurgical nerve root canal widening without fusion for lumbosacral intervertebral foraminal stenosis: technical notes and early results. Spinal Cord 1996; 34: 644-650.

4 Jabre A, Spatz EL. Far lateral lumbar disc herniation. Diagnosis and treatment by inferior facetectomy approach. J Neurol Orthop Med Surg 1996; 17: $18-24$

5 Epstein NE. Different surgical approaches to far lateral lumbar disc herniations. J Spinal Disord 1995; 8: $383-394$.

6 Epstein NE, Epstein JA, Carras R, Hyman RA. Far lateral lumbar disc herniations and associated structural abnormalities: an evaluation in 60 patients of the comparative value of CT, MRI, and myelo-CT in diagnosis and management. Spine 1990; 15: $534-539$.

7 Reulen HJ, Pfaundler S, Ebelling U. The lateral microsurgical approach to the 'extracanalicular' lumbar disc herniation. Acta Neurochir (Wien) 1987; 84: 64-67.

8 Lee CK, Wolfgang R, William G. Lateral lumbar spinal canal stenosis: classification, pathologic anatomy and surgical decompression. Spine 1988; 13: 313-320.

9 Jane JA et al. A neurosurgical approach to far-lateral disc herniation: technical note. J Neurosurg 1990; 72: $143-144$.

10 Stephens MM, Evans JH, O'Brien JP. Lumbar intervertebral foramens: an in vitro study of their shape in relation to intervertebral disc pathology. Spine 1991; 16: 525-529.

11 Major NM, Helmes CA. Central and foraminal stenosis of the lumbar spine. Neuroimaging Clin North Am 1993; 3: 557-566.

12 Autrique et al. Le traitement chirurgical des hernies discales foraminales lombaires. Intérêsts et indications de la voie combinée interlaminaire et extraarticulaire. J Chir (Paris) 1989; 126: 338 - 343 .

13 Garrido E, Connaughton PN. Unilateral facetectomy approach for lateral lumbar disc herniation. J Neurosurg 1991; 74: $754-$ 756.

14 Marron JC et al. Diagnosis and microsurgical approach to farlateral disc herniation in the lumbar spine. J Neurosurg 1990; 72: $378-382$.

15 Darden BV II et al. Far lateral disc herniation treated by microscopic fragment excision: techniques and results. Spine 1995; 20: $1500-1505$.

16 Fankhauser $\mathrm{H}$, de Tribolet N. Extraforaminal approach for extreme lateral lumbar disc herniation. In: Torrens MJ, Dickson RA (eds). Operative Spinal Surgery. Churchill Livingstone: Edinburgh, 1991, pp 145-160. 